\title{
Feature Articles in Blog
}

\author{
Anggi Cecilia Safaningrum ${ }^{1\left(^{*}\right)}$ \\ ${ }^{1}$ Jupenizer Institute, Raffles Hills, Cluster Beauty X, no. 6, Jakarta Timur, 13720, Indonesia \\ (*) asafaningrum@gmail.com
}

\begin{abstract}
Feature articles are generally the stories in newspapers and magazines other than straight news stories, editorials, or advertising. In addition, because of their human interest, they attempt to involve the reader emotionally. We have chosen feature articles from Alobatnic's blog that will provide a resource for those who are new to explorations of feature article in blogger, key terminology, and brief definitions.
\end{abstract}

Keywords : feature articles; online publising;

A feature article gives information of human interest. Feature articles are generally the stories in newspapers and magazines other than straight news stories, editorials, or advertising. In addition, because of their human interest, they attempt to involve the reader emotionally. The feature article does not have the inverted pyramid organization of the "hard" news article. Furthermore, features do not become dated as quickly as "hard" news stories. A good feature article is often interesting to read a year after it was written. It is more similar to fiction because it tells a story. For example, a news story about the Prime Minister might be about what is happening in Cabinet or perhaps which laws are under consideration in the Diet. A feature article, on the other hand, would perhaps look at the Prime Minister's leisure activities or tell the story of some important formative incident that took place in his youth.

\section{Common types of Feature Articles \\ The Human Interest Feature}

A human interest feature takes something currently of interest in society and connects it to something interesting in the life of an ordinary person. For example, a feature article on email might show us e-mail is changing human relationships at work and at home.

\section{The Personality Feature}

A human interest feature may develop into a personality feature which looks at a person's life in more depth. The person may be well known or completely unknown, but he/she will have done something of interest to others. For example, Rick Hansen, a wheelchair athlete, travelled the equivalent of the distance around the world to raise money for spinal cord injury research. His life has been the subject of many personality feature articles. A personality feature may be tragic or inspire us because of the heroics of the subject.

\section{The How-To Feature}

The how-to feature describes how to do or build something. For example, how to organize your desk for efficienty, or how to clean your house in twenty minutes.

\section{The Past Events Feature}

The past events feature looks at historical events, but approaches them from the angle of human interest. For example, in remembering the 1964 Tokyo Olympics, rather than look only at the economic or political facets of the story, a feature article might look at how the Tokyo Olympics affected one person's life in particular.

\section{The News Feature}

The news feature takes a current news story as its start but then investigates how the news affects the lives of average people. For example, how the employees at a small company whose boss has been killed in a plane crash, continue running the company. 


\section{Writing and Organizing a Feature Article}

- Choose which type of feature article you want to write. Then choose which emotion you want to appeal to in your readers.

- Decide which method of organization will be most effective for your story: order of importance or chronological order.

- Decide how you want to lead into the story. Start with a sentence that catches the attention of your readers and makes them want to finish reading the story.

- Use the techniques of story writing to help develop the human interest in your article.

- Conclude your article in the same way as you would a short story. Be sure to bring together all the loose ends.

- Create a headline that will catch your readers' attention.

\section{Feature Article Checklist}

- Is my research complete and accurate?

- Does my opening catch the readers' attention?

- Is my article interesting throughout?

- How have I organized my article? Is it logical?

- Have I used a variety of sentence lengths and styles?

- Have I included transitions to improve the flow and coherence?

- Have I used fresh, effective words?

- Have I removed all unnecessary words?

- Do I tell an interesting story? 


\section{REFERENCES}

Louisa, Grace Natalie, \& Setiawan, Adib Rifqi. (2019, April 12). Q \& A. Grace Natalie Louisa. [interview]. Open Science Framework. DOI: https://doi.org/10.31219/osf.io/aq5kg

Setiawan, Adib Rifqi, \& Amatullah. (2016, October 01). Berkarya Sebagai Diaspora: Ulas Kilas Nadirsyah Hosen. Alobatnic.blogspot.com. URL: https://alobatnic.blogspot.com/2016/10/berkarya-sebagai-diaspora.html

Setiawan, Adib Rifqi, \& Louisa, Grace Natalie. (2018, December 11). Keadilan Untuk Semua, Keadilan Untuk Perempuan Indonesia. Alobatnic.blogspot.com. URL: https://alobatnic.blogspot.com/2018/12/keadilan-untuk-semua-keadilan-untuk.html

Setiawan, Adib Rifqi, \& Louisa, Grace Natalie. (2019, February 11). Politik Akal Sehat, Politik Kaum Muda Alobatnic.blogspot.com. URL: https://alobatnic.blogspot.com/2019/02/musuh-utama-persatuan-indonesia.html

Setiawan, Adib Rifqi, \& Louisa, Grace Natalie. (2019, January 11). Politik Akal Sehat, Politik Kaum Muda Alobatnic.blogspot.com. URL: https://alobatnic.blogspot.com/2019/01/politik-akal-sehat-politik-kaum-muda.html

Setiawan, Adib Rifqi, \& Ma'munah, Malikatul. (2015, May 27). Nun: bahadur menghibur melantan pengharapan. Alobatnic.blogspot.com. URL: https://alobatnic.blogspot.com/2015/05/emha.html

Setiawan, Adib Rifqi. (2013, August 26). Bathari Ekonomi: tak langsir dirisak penyinyir. Alobatnic.blogspot.com. URL: https://alobatnic.blogspot.com/2013/08/sri.html

Setiawan, Adib Rifqi. (2013, July 15). Menjilati Yuli: tegap menghibur walau dalam tangis. Alobatnic.blogspot.com. URL: https://alobatnic.blogspot.com/2013/07/yuli.html

Setiawan, Adib Rifqi. (2013, March 10). Kirana Tanpa Sirna: sepanjang menggelinjang memahat maslahat. Alobatnic.blogspot.com. URL: https://alobatnic.blogspot.com/2013/03/turoichan.html

Setiawan, Adib Rifqi. (2013, November 06). Cendekiawan Picisan: melintang lintang intan terabaikan. Alobatnic.blogspot.com. URL: https://alobatnic.blogspot.com/2013/11/yohanes.html

Setiawan, Adib Rifqi. (2013, October 17). Pangeran Ternistakan: kontroversi serta ambisi tiada henti. Alobatnic.blogspot.com. URL: https://alobatnic.blogspot.com/2013/10/prabowo.html

Setiawan, Adib Rifqi. (2014, July 17). Karena Lelaki Bukan Pengecut: driven by you tie your mother down. Alobatnic.blogspot.com. URL: https://alobatnic.blogspot.com/2014/07/brian.html

Setiawan, Adib Rifqi. (2014, June 18). Glam Male: kirana bunga manusia biasa. Alobatnic.blogspot.com. URL: https://alobatnic.blogspot.com/2014/06/andra.html

Setiawan, Adib Rifqi. (2014, May 26). Risalah Kasidah: bayang bayang satu sisi restoe boemi. Alobatnic.blogspot.com. URL: https://alobatnic.blogspot.com/2014/05/dhani.html

Setiawan, Adib Rifqi. (2014, September 05). Popularitas adalah Tuhan: memberikan penghiburan, menumbuhkan pengharapan. Alobatnic.blogspot.com. URL: https://alobatnic.blogspot.com/2014/09/farrokh.html

Setiawan, Adib Rifqi. (2015, June 02). Melantan Warisan Kaisar Telantar: telantar, telantar, telantar, terlantan. Alobatnic.blogspot.com. URL: https://alobatnic.blogspot.com/2015/06/ibrahim.html

Setiawan, Adib Rifqi. (2015, May 11). Butcah Mbeling: the mad one's behavior in motion. Alobatnic.blogspot.com. URL: https://alobatnic.blogspot.com/2015/05/butcahmbeling-richard-phillips-feynman.html 
Setiawan, Adib Rifqi. (2016, February 11). Mike Shinoda. Alobatnic.blogspot.com. URL: https://alobatnic.blogspot.com/2016/02/mike-shinoda.html

Setiawan, Adib Rifqi. (2016, February 17). PARIS: nothing in this world turn you on. Alobatnic.blogspot.com. URL: https://alobatnic.blogspot.com/2016/02/paris.html

Setiawan, Adib Rifqi. (2016, January 22). Ionia: lingkungan pemula gelora kajian keilmuan. Alobatnic.blogspot.com. URL: https://alobatnic.blogspot.com/2016/01/ionia.html

Setiawan, Adib Rifqi. (2016, July 23). Kamu Dalam aku: memuja yang dicinta, membela yang dinista. Alobatnic.blogspot.com. URL: https://alobatnic.blogspot.com/2016/07/bom.html

Setiawan, Adib Rifqi. (2016, November 17). K2: mengenang sahabat yang hebat. Alobatnic.blogspot.com. URL: https://alobatnic.blogspot.com/2016/11/kaka.html

Setiawan, Adib Rifqi. (2017, April 29). My Way Rhapsody!: Islam Aries-Blackjack, Is Islam?. Alobatnic.blogspot.com. URL: https://alobatnic.blogspot.com/2017/04/islam.html

Setiawan, Adib Rifqi. (2017, February 15). Butcah Chuniez: Puzzle Persinggungan Perjalanan dengan Eny Rochmwati Octaviani. Alobatnic.blogspot.com. URL: https://alobatnic.blogspot.com/2017/02/thata.html

Setiawan, Adib Rifqi. (2017, February 24). Penerapan Pendekatan Saintifik Untuk Melatihkan Literasi Saintifik Dalam Domain Kompetensi Pada Topik Gerak Lurus Di Sekolah Menengah Pertama. Undergraduate Thesis. Universitas Pendidikan Indonesia. URL: http://repository.upi.edu/29074/

Setiawan, Adib Rifqi. (2017, July 15). Hope, Faith, Love: A Brief Story of Yuli Rahmawati. Alobatnic.blogspot.com. URL: https://alobatnic.blogspot.com/2017/07/hope-faithlove.html

Setiawan, Adib Rifqi. (2017, June 03). Meniti Ilmuwati: catatan peran perempuan dalam pembahasan alam. Alobatnic.blogspot.com. URL: https://alobatnic.blogspot.com/2017/06/meniti-ilmuwati-scholaristi.html

Setiawan, Adib Rifqi. (2017, March 23). Guru yang Menyapih: tak melayang dipuji, tak tumbang dicaci. Alobatnic.blogspot.com. URL: https://alobatnic.blogspot.com/2017/03/nong.html

Setiawan, Adib Rifqi. (2017, May 21). Itz Spring Voice: andai aku tak mengaguminya .... Alobatnic.blogspot.com. URL: https://alobatnic.blogspot.com/2017/05/itz.html

Setiawan, Adib Rifqi. (2017, September 22). Mengonstruksi rancangan soal domain kompetensi literasi saintifik siswa smp kelas viii pada topik gerak lurusMengonstruksi rancangan soal domain kompetensi literasi saintifik siswa smp kelas viii pada topik gerak lurus. Wahana Pendidikan Fisika (WaPFi), 2(2), 44-8. URL: http://ejournal.upi.edu/index.php/WapFi/article/view/8277

Setiawan, Adib Rifqi. (2018, April 01). Ternyata Majalah SANTRI Masih Terbit!: catatan pribadi mengenai penerbitan Majalah SANTRI edisi (10) April 2018. Alobatnic.blogspot.com. URL: https://alobatnic.blogspot.com/2018/04/ternyatamajalah-santri-masih-terbit.html

Setiawan, Adib Rifqi. (2018, April 02). Rosa Amalia Iqony: paduan yakin diri dan rendah hati. Alobatnic.blogspot.com. URL: https://alobatnic.blogspot.com/2018/04/rosaamalia-iqony.html

Setiawan, Adib Rifqi. (2018, April 04). Busana: pemantas raga, pelaras jiwa. Alobatnic.blogspot.com. URL: https://alobatnic.blogspot.com/2018/04/busana.html

Setiawan, Adib Rifqi. (2018, April 10). Busana. Majalah SANTRI, pp. 26-7. URL: https://issuu.com/majalahsantri/docs/majalah_santri_8/28

Setiawan, Adib Rifqi. (2018, April 10). Eny Rochmawati Octaviani. Majalah SANTRI, pp. 26-7. URL: https://issuu.com/majalahsantri/docs/majalah_santri_8/17 
Setiawan, Adib Rifqi. (2018, April 10). Eny Rochmawati Octaviani: memberikan hiburan, menyuntikkan harapan. Alobatnic.blogspot.com. URL:

https://alobatnic.blogspot.com/2018/04/eny-rochmawati-octaviani.html

Setiawan, Adib Rifqi. (2018, April 10). Rosa Amalia Iqony. Majalah SANTRI, pp. 46. URL: https://issuu.com/majalahsantri/docs/majalah_santri_8/48

Setiawan, Adib Rifqi. (2018, April 11). Risalah Rihlah (Catatan Penjelajahan): melestarikan tradisi bermadzhab. Alobatnic.blogspot.com. URL: https://alobatnic.blogspot.com/2018/04/risalah- rihlah.html

Setiawan, Adib Rifqi. (2018, August 04). Debut Mengajar Biologi: mengenali diri, melantan lingkungan. Alobatnic.blogspot.com. URL: https://alobatnic.blogspot.com/2018/08/debut-mengajar-biologi.html

Setiawan, Adib Rifqi. (2018, August 11). Biology : My Story. Alobatnic.blogspot.com. URL: https://alobatnic.blogspot.com/2018/08/biology-my-story.html

Setiawan, Adib Rifqi. (2018, December 12). A Brief Note of Science Education Development in Indonesia. Alobatnic.blogspot.com. URL: https://alobatnic.blogspot.com/2018/12/a-brief-note-of-science-education.html

Setiawan, Adib Rifqi. (2018, December 17). Grace Natalie, PSI, Poligami. Alobatnic.blogspot.com. URL: https://alobatnic.blogspot.com/2018/12/grace-nataliepsi-poligami.html

Setiawan, Adib Rifqi. (2018, December 21). Irma Rahma Suwarma. Alobatnic.blogspot.com. URL: https://alobatnic.blogspot.com/2018/12/irma-rahma-suwarma.html

Setiawan, Adib Rifqi. (2018, December 21). Upaya Melatih Literasi Saintifik melalui Pembelajaran Ilmu Pengetahuan Alam (IPA). Alobatnic.blogspot.com. URL: https://alobatnic.blogspot.com/2018/12/upaya-melatih-literasi-saintifik.html

Setiawan, Adib Rifqi. (2018, December 24). Madrasah Tasywiquth Thullab Salafiyyah (TBS) Kudus. Alobatnic.blogspot.com. URL:

https://alobatnic.blogspot.com/2018/12/madrasah-tasywiquth-thullab-salafiyyah-tbskudus.html

Setiawan, Adib Rifqi. (2018, December 28). Garis Besar Haluan Matematika: towards a natural system of the mathematical world: proposal for the principle of least action in teaching mathematics. Alobatnic.blogspot.com. URL: https://alobatnic.blogspot.com/2018/12/garis-besar-haluan-matematika.html

Setiawan, Adib Rifqi. (2018, December 31). Mabādī 'Asyroh Fisika. Alobatnic.blogspot.com. URL: https://alobatnic.blogspot.com/2018/12/mabadi-asyroh-fisika.html

Setiawan, Adib Rifqi. (2018, February 08). Sekilas Mengelilingi Luas Geometri: Mengingat René Descartes, Mengenang Muḥammad al-Ghazālī. Alobatnic.blogspot.com. URL: https://alobatnic.blogspot.com/2018/02/sekilas-mengelilingi-luas-geometri.html

Setiawan, Adib Rifqi. (2018, February 27). Maryam Musfiroh: An Educator for Our Time. Alobatnic.blogspot.com. URL: https://alobatnic.blogspot.com/2018/02/maryammusfiroh.html

Setiawan, Adib Rifqi. (2018, January 01). Pantat Perekat Umat: Modal Erotic Zaskia Gotik. Alobatnic.blogspot.com. URL: https://alobatnic.blogspot.com/2018/01/pantat-perekatumat.html

Setiawan, Adib Rifqi. (2018, January 05). This is Venice: tak lelah meniti tatanan, tak jengah menata titian. Alobatnic.blogspot.com. URL: https://alobatnic.blogspot.com/2018/01/islam-aries-blackjack.html

Setiawan, Adib Rifqi. (2018, January 08). Islām Aries-Blackjack, is Islām?. Alobatnic.blogspot.com. URL: https://alobatnic.blogspot.com/2018/01/islam-ariesblackjack.html 
Setiawan, Adib Rifqi. (2018, January 11). Roman (Bukan) Picisan: tak lelah meniti tatatan, tak jengah menata titian. Alobatnic.blogspot.com. URL:

https://alobatnic.blogspot.com/2018/01/islam-aries-blackjack.html

Setiawan, Adib Rifqi. (2018, January 15). Clara Ng: an author for our time.

Alobatnic.blogspot.com. URL: https://alobatnic.blogspot.com/2018/01/islam-ariesblackjack.html

Setiawan, Adib Rifqi. (2018, January 16). Amazing Grace: Satu Sisi Religi Grace Natalie. Alobatnic.blogspot.com. URL: https://alobatnic.blogspot.com/2018/01/amazinggrace.html

Setiawan, Adib Rifqi. (2018, January 17). Laila Fariha Zein (a.k.a. Uus atau Febi): penyuluh peluh, peluluh keluh, peluruh keruh. Alobatnic.blogspot.com. URL: https://alobatnic.blogspot.com/2018/01/laila-fariha-zein.html

Setiawan, Adib Rifqi. (2018, January 18). Dari Fallen Menjadi Vallen: Fall in Love Vallen in Love in Valentine. Alobatnic.blogspot.com. URL: https://alobatnic.blogspot.com/2018/01/via-vallen.html

Setiawan, Adib Rifqi. (2018, January 19). Aisha: rebel heart living for love. Alobatnic.blogspot.com. URL: https://alobatnic.blogspot.com/2018/01/aisha.html

Setiawan, Adib Rifqi. (2018, January 26). Remy Sylado 23761: konsisten dengan seribu kemuliaan. Alobatnic.blogspot.com. URL: https://alobatnic.blogspot.com/2018/01/remy-sylado-23761.html

Setiawan, Adib Rifqi. (2018, July 03). Crazy Rhapsody: deep in my heart. Alobatnic.blogspot.com. URL: https://alobatnic.blogspot.com/2018/07/crazyrhapsody.html

Setiawan, Adib Rifqi. (2018, July 22). Paul DeHart Hurd: menjadi-jadi dari biologi. Alobatnic.blogspot.com. URL: https://alobatnic.blogspot.com/2018/07/paul-deharthurd.html

Setiawan, Adib Rifqi. (2018, June 13). Perjuangan Angan Perempuan: Obrolan dengan Grace Natalie Louisa. Alobatnic.blogspot.com. URL: https://alobatnic.blogspot.com/2018/06/perjuangan-angan-perempuan.html

Setiawan, Adib Rifqi. (2018, June 13). Perjuangan Angan Perempuan: Obrolan dengan Grace Natalie Louisa. Alobatnic.blogspot.com. URL: https://alobatnic.blogspot.com/2018/06/perjuangan-angan-perempuan.html

Setiawan, Adib Rifqi. (2018, June 15).Come Back Bom! [컴백봄!]. Alobatnic.blogspot.com. URL: https://alobatnic.blogspot.com/2018/06/come-back-bom.html

Setiawan, Adib Rifqi. (2018, June 20).al-Munqidz min al-Dholāl karya Abū Ḥāmid Muhammad al-Ghozāiñ: pembebas dari kesalahan menuju kebenaran berlandaskan keilmuan. Alobatnic.blogspot.com. URL: https://alobatnic.blogspot.com/2018/06/almunqidz-min-al-dholal-karya-abu-hamid-muhammad-al-ghazali.html

Setiawan, Adib Rifqi. (2018, June 22).Fakhrun: a brief friendship (5 November 11 - 2 Agustus 2014). Alobatnic.blogspot.com. URL: https://alobatnic.blogspot.com/2018/06/fakhrun.html

Setiawan, Adib Rifqi. (2018, June 27).Drama Korea. Alobatnic.blogspot.com. URL: https://alobatnic.blogspot.com/2018/06/drama-korea.html

Setiawan, Adib Rifqi. (2018, June 28).Dewi Perssik: dari sensasi ke sensasi hingga daya menghadapi suami. Alobatnic.blogspot.com. URL: https://alobatnic.blogspot.com/2018/06/dewi-perssik.html

Setiawan, Adib Rifqi. (2018, March 01). Ki Oza Kioza: A Rain Shine Made in Indonesia. Alobatnic.blogspot.com. URL: https://alobatnic.blogspot.com/2018/03/roza-lailatulfitria-oza-kioza.html 
Setiawan, Adib Rifqi. (2018, March 05). Breast Capital: Konsep Baru Duo Serigala, Modal Erotis, dan Payudara Perempuan. Alobatnic.blogspot.com. URL: https://alobatnic.blogspot.com/2018/03/breastcapital.html

Setiawan, Adib Rifqi. (2018, March 08). Apresiasi Semadyana pada YoonA: Ketika YoonA mampir ke Parc des Princes. Alobatnic.blogspot.com. URL: https://alobatnic.blogspot.com/2018/03/apresiasi-semadyana-pada-yoona.html

Setiawan, Adib Rifqi. (2018, March 09). Venice Min: yakin diri dan rendah hati memanfaatkan kecantikan. Alobatnic.blogspot.com. URL: https://alobatnic.blogspot.com/2018/03/venice-min.html

Setiawan, Adib Rifqi. (2018, March 24). Hard to Say Happy Birthday Park Bom. Alobatnic.blogspot.com. URL: https://alobatnic.blogspot.com/2018/03/hard-to-sayhappy-birthday-park-bom.html

Setiawan, Adib Rifqi. (2018, March 30). Menelusuri Nama Maryam: anyam sanggam, anyam azam. Alobatnic.blogspot.com. URL: https://alobatnic.blogspot.com/2018/03/menelusuri-nama-maryam.html

Setiawan, Adib Rifqi. (2018, May 05). Girls, Love, War: A Brief Story of Rahwayana. Alobatnic.blogspot.com. URL: https://alobatnic.blogspot.com/2018/05/rahwayana.html

Setiawan, Adib Rifqi. (2018, May 11). Mathematics: to write nor not to write. Alobatnic.blogspot.com. URL: https://alobatnic.blogspot.com/2018/05/mathematics.html

Setiawan, Adib Rifqi. (2018, May 19). Máthēmatnic: catatan perjalanan. Alobatnic.blogspot.com. URL: https://alobatnic.blogspot.com/2018/05/mathematnic.html

Setiawan, Adib Rifqi. (2018, May 19). Máthēmatnic: catatan perjalanan. Alobatnic.blogspot.com. URL: https://alobatnic.blogspot.com/2018/05/mathematnic.html

Setiawan, Adib Rifqi. (2018, May 20). Manunggaling Meyda Sefira-Hujan Safir. Alobatnic.blogspot.com. URL: https://alobatnic.blogspot.com/2018/05/manunggalingmeyda-sefira-hujan-safir.html

Setiawan, Adib Rifqi. (2018, November 10). Pain Man or Fine Man?: Apa sih yang Istimewa dari Richard Phillips Feynman?. Alobatnic.blogspot.com. URL: https://alobatnic.blogspot.com/2018/11/richard-phillips-feynman-pain-man-or-fineman.html

Setiawan, Adib Rifqi. (2018, November 13). Surely You're Joking, Mr. Feynman!: Adventures of a Curious Character. Alobatnic.blogspot.com. URL: https://alobatnic.blogspot.com/2018/11/surely-youre-joking-mr-feynman.html

Setiawan, Adib Rifqi. (2018, November 14). Six Not-So-Amazing Video. Alobatnic.blogspot.com. URL: https://alobatnic.blogspot.com/2018/11/six-not-soamazing-video.html

Setiawan, Adib Rifqi. (2018, November 16). Segitiga Sama Cinta: who is the queen of the sciences: mathematics or biology?. Alobatnic.blogspot.com. URL: https://alobatnic.blogspot.com/2018/11/segitiga-sama-cinta.html

Setiawan, Adib Rifqi. (2018, November 29). Risalah Rihlah. Alobatnic.blogspot.com. URL: https://alobatnic.blogspot.com/2018/11/risalah-rihlah.html

Setiawan, Adib Rifqi. (2018, October 09). Bergerak Menuju Tak Tentu: risalah rihlah khidmah 'ilmiyyah. Alobatnic.blogspot.com. URL: https://alobatnic.blogspot.com/2018/10/bergerak-menuju-tak-tentu.html

Setiawan, Adib Rifqi. (2018, October 18). Ketika Ibrōhīm Mengajak untuk Mengamati Alam: dari dorongan penasaran sampai gairah berserah. Alobatnic.blogspot.com. URL: 
https://alobatnic.blogspot.com/2018/10/ketika-ibrohim-mengajak-untukmengamati.html

Setiawan, Adib Rifqi. (2018, October 25). Hukum Alam, Kehendak Bebas, Perilaku Manusia. Alobatnic.blogspot.com. URL: https://alobatnic.blogspot.com/2018/10/hukum-alamkehendak-bebas-perilaku-manusia.html

Setiawan, Adib Rifqi. (2018, October 25). Into the New World (다시 만난 세계): proposal for the principle of least action in the simple life. Alobatnic.blogspot.com. URL: https://alobatnic.blogspot.com/2018/10/into-the-new-world.html

Setiawan, Adib Rifqi. (2018, September 16). Lola Zieta Azelien: paduan yakin diri dan rendah hati memanfaatkan kecantikan. Alobatnic.blogspot.com. URL: https://alobatnic.blogspot.com/2018/09/lola-zieta-azelien.html

Setiawan, Adib Rifqi. (2018, September 25). Peran Biologi dalam Mendukung Pelaksanaan Jihād. Alobatnic.blogspot.com. URL: https://alobatnic.blogspot.com/2018/09/peranbiologi-dalam-mendukung-pelaksanaan-jihad.html

Setiawan, Adib Rifqi. (2018, September 25). Peran Biologi dalam Mendukung Pelaksanaan Jihād. Alobatnic.blogspot.com. URL: https://alobatnic.blogspot.com/2018/09/peranbiologi-dalam-mendukung-pelaksanaan-jihad.html

Setiawan, Adib Rifqi. (2018, September 30). Karen Armstrong: there is a rainbow in Islām. Alobatnic.blogspot.com. URL: https://alobatnic.blogspot.com/2018/09/karenarmstrong.html

Setiawan, Adib Rifqi. (2019, April 1). Maryam Musfiroh: An Educator for Our Time. INARxiv. DOI: https://doi.org/10.31227/osf.io/qn39r

Setiawan, Adib Rifqi. (2019, April 10). Laila Isrofatun Nahdiah. Open Science Framework. DOI: https://doi.org/10.31219/osf.io/kj8tz

Setiawan, Adib Rifqi. (2019, April 10). Risalah Ilmiyah: Adventures with a Curious Leader. Open Science Framework. DOI: https://doi.org/10.31219/osf.io/jzbg5

Setiawan, Adib Rifqi. (2019, April 11). Godly Nationalism: Tribute to Santri Scholar Press. Open Science Framework. DOI: https://doi.org/10.31219/osf.io/dfxga

Setiawan, Adib Rifqi. (2019, April 11). Melantan Warisan Kaisar Telantar. Open Science Framework. DOI: https://doi.org/10.31219/osf.io/jxpnc

Setiawan, Adib Rifqi. (2019, April 12). Q \& A. Grace Natalie Louisa. [interview]. Alobatnic.blogspot.com. URL: https://alobatnic.blogspot.com/2019/04/grace-natalielouisa.html

Setiawan, Adib Rifqi. (2019, April 15). Breast Capital. Open Science Framework. DOI: https://doi.org/10.31219/osf.io/vkq6x

Setiawan, Adib Rifqi. (2019, April 15). Pantat Perekat Umat. Open Science Framework. DOI: https://doi.org/10.31219/osf.io/m5qdv

Setiawan, Adib Rifqi. (2019, April 15). Rosa Amalia Iqony: How Muslimah Use Her Erotic Capital?. Open Science Framework. DOI: https://doi.org/10.31219/osf.io/x9k23

Setiawan, Adib Rifqi. (2019, April 15). Venice Min: A Model for Our Time. Open Science Framework. DOI: https://doi.org/10.31219/osf.io/64jfs

Setiawan, Adib Rifqi. (2019, April 18). Jessica Jung: three decades of the modern-classic aesthetic sequencing. Open Science Framework. DOI: https://doi.org/10.31219/osf.io/8vjpb

Setiawan, Adib Rifqi. (2019, April 24). Bercerita Cerita Clara. Open Science Framework. DOI: https://doi.org/10.31219/osf.io/4jhkt

Setiawan, Adib Rifqi. (2019, April 24). Clara Ng: A Brief Journey of A Remarkable Female for Our Time. LIS Scholarship Archive. DOI: https://doi.org/10.31229/osf.io/ktrhm 
Setiawan, Adib Rifqi. (2019, April 24). Clara Ng: Un Bref Voyage d'Une Femme Remarquable pour Notre Temps. Open Science Framework. DOI: https://doi.org/10.31219/osf.io/tn93q

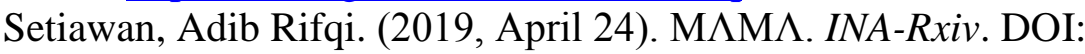
https://doi.org/10.31227/osf.io/73wc5

Setiawan, Adib Rifqi. (2019, April 24). Q \& A. Lisa Randall. [interview]. Open Science Framework. DOI: https://doi.org/10.31219/osf.io/sjfxn

Setiawan, Adib Rifqi. (2019, April 25). OZA KIOZA. SocArXiv. DOI: https://doi.org/10.31235/osf.io/8mqwk

Setiawan, Adib Rifqi. (2019, April 25). Paris, Not Française: A Brief Journey of The Simple Person. Open Science Framework. DOI: https://doi.org/10.31219/osf.io/w5qh3

Setiawan, Adib Rifqi. (2019, April 26). An Interview with Venice Min. Alobatnic.blogspot.com. URL: https://alobatnic.blogspot.com/2019/04/an-interviewwith-venice-min.html

Setiawan, Adib Rifqi. (2019, April 26). Curahan Hati untuk Teman-Teman Santri. Alobatnic.blogspot.com. URL: https://alobatnic.blogspot.com/2019/04/curahan-hatiuntuk-teman-teman-santri.html

Setiawan, Adib Rifqi. (2019, April 26). Meniti Ilmuwati. Open Science Framework. DOI: https://doi.org/10.31219/osf.io/6x3af

Setiawan, Adib Rifqi. (2019, April 26). Panduan Penulisan Artikel. Alobatnic.blogspot.com. URL: https://alobatnic.blogspot.com/2019/04/panduan-penulisan-artikel.html

Setiawan, Adib Rifqi. (2019, April 26). Panduan Penulisan Artikel Akademik. Open Science Framework. DOI: https://doi.org/10.31219/osf.io/2f7pr

Setiawan, Adib Rifqi. (2019, April 26). Q \& A. Lisa Randall. [interview]. Alobatnic.blogspot.com. URL: https://alobatnic.blogspot.com/2019/04/q-lisa-randallinterview.html

Setiawan, Adib Rifqi. (2019, April 27). Kurikulum Lokal Madrasah Tasywiquth Thullab Salafiyyah (TBS) Kudus. Open Science Framework. DOI: https://doi.org/10.31219/osf.io/dcvum

Setiawan, Adib Rifqi. (2019, April 29). Kehidupan Perempuan Etnis Tionghoa pada Orde Militeristik dalam 'Dimsum Terakhir' karya Clara Ng. LIS Scholarship Archive. DOI: https://doi.org/10.31229/osf.io/s9dej

Setiawan, Adib Rifqi. (2019, April 3). A Leader for Our Time: Ulasan Biografi Grace Natalie Louisa. INA-Rxiv. DOI: https://doi.org/10.31227/osf.io/73y9u

Setiawan, Adib Rifqi. (2019, April 3). Amazing Grace: Sisi Religi Grace Natalie Louisa Ketua Umum Partai Solidaritas Indonesia. INA-Rxiv. DOI: https://doi.org/10.31227/osf.io/y9krs

Setiawan, Adib Rifqi. (2019, April 3). Grace Natalie, PSI, Poligami: Sisi Feminis Grace Natalie Louisa Ketua Umum Partai Solidaritas Indonesia. INA-Rxiv. DOI: https://doi.org/10.31227/osf.io/s7qzb

Setiawan, Adib Rifqi. (2019, April 3). Mabādī 'Asyroh Fisika. INA-Rxiv. DOI: https://doi.org/10.31227/osf.io/jey4k

Setiawan, Adib Rifqi. (2019, April 3). Perjuangan Angan Perempuan: Sisi Politik Grace Natalie Louisa Ketua Umum Partai Solidaritas Indonesia. INA-Rxiv. DOI: https://doi.org/10.31227/osf.io/5akw3

Setiawan, Adib Rifqi. (2019, January 05). We Broke Up, We Rising Up: From 2015 MAMA to 2NE1's breakup to BL $\Lambda$ DKPIИK's riseup. Alobatnic.blogspot.com. URL: https://alobatnic.blogspot.com/2019/01/we-broke-up.html 
Setiawan, Adib Rifqi. (2019, January 14). Basic Islamic Studies for Children: basic islamic studies for children from my traditional islamic perspective. Alobatnic.blogspot.com. URL: https://alobatnic.blogspot.com/2019/01/basic-islamic-studies-for-children.html

Setiawan, Adib Rifqi. (2019, March 10). Busana. INA-Rxiv. DOI: https://doi.org/10.31227/osf.io/mzca9

Setiawan, Adib Rifqi. (2019, March 10). Eny Rochmawati Octaviani. INA-Rxiv. DOI: https://doi.org/10.31227/osf.io/t296z

Setiawan, Adib Rifqi. (2019, March 10). Nong Darol Mahmada: Guru yang Menyapih. INARxiv. DOI: https://doi.org/10.31227/osf.io/8nerv

Setiawan, Adib Rifqi. (2019, March 10). Rosa Amalia Iqony. INA-Rxiv. DOI: https://doi.org/10.31227/osf.io/rymxt

Setiawan, Adib Rifqi. (2019, March 11). Tak Melayang Dipuji, Tak Tumbang Dicaci: Kajian Biografi Oza Kioza. INA-Rxiv. DOI: https://doi.org/10.31227/osf.io/9a8z7

Setiawan, Adib Rifqi. (2019, March 14). Guru yang Menyapih: Biografi Pribadi Nong Darol Mahmada. INA-Rxiv. DOI: https://doi.org/10.31227/osf.io/ky4c2

Setiawan, Adib Rifqi. (2019, March 19). Tak Melayang Dipuji, Tak Tumbang Dicaci: Kajian Biografi Oza Kioza. INA-Rxiv. DOI: https://doi.org/10.31227/osf.io/vwe4g

Setiawan, Adib Rifqi. (2019, March 21). Bergerak Menuju Tak Tentu. INA-Rxiv. DOI: https://doi.org/10.31227/osf.io/4x9kb

Setiawan, Adib Rifqi. (2019, March 27). Integrasi Kaidah Fiqih dalam Pembelajaran Ekologi. INA-Rxiv. DOI: https://doi.org/10.31227/osf.io/24mzd

Setiawan, Adib Rifqi. (2019, March 29). Máthēmatnic: Pengalaman Memandu Pembelajaran Matematika. INA-Rxiv. DOI: https://doi.org/10.31227/osf.io/fx7uw

Setiawan, Adib Rifqi. (2019, May 3). Ekspresi Perempuan dalam 'Kill This Love' dari BLACKPINK. INA-Rxiv. DOI: https://doi.org/10.31227/osf.io/rh67u

Setiawan, Adib Rifqi. (2019, May 3). How Girls Generation Use Erotic Capital?. INA-Rxiv. DOI: https://doi.org/10.31227/osf.io/xkrzw

Setiawan, Adib Rifqi. (2019, May 3). Sisi Feminis Perempuan Korea Selatan dalam album 'To Anyone' dari 2NE1. INA-Rxiv. DOI: https://doi.org/10.31227/osf.io/jyt43

Setiawan, Adib Rifqi., \& Min, Venice. (2019, April 24). An Interview with Venice Min. Open Science Framework. DOI: https://doi.org/10.31219/osf.io/yj5b8

Setiawan, Adib Rifqi., \& Musfiroh, Maryam. (2019, March 21). Pendidikan Karakter: Akhlak, Adab, Moral dan Nilai. INA-Rxiv. DOI: https://doi.org/10.31227/osf.io/x8syc

Setiawan, Adib Rifqi., \& Octaviani, Eny Rochmawati. (2019, April 25). Yoona: A Brief Journey of An Entertainer for Our Time. Open Science Framework. DOI: https://doi.org/10.31219/osf.io/e4bhd

Zein, Laila Fariha, \& Setiawan, Adib Rifqi. (2019, April 9). Julia Perez: une authentique modèle. INA-Rxiv. DOI: https://doi.org/10.31227/osf.io/br9vt

Zein, Laila Fariha, \& Setiawan, Adib Rifqi. (2019, April 9). Kecantikan Perempuan dalam As If It's Your Last karya BLACKPINK. INA-Rxiv. DOI: https://doi.org/10.31227/osf.io/pfrz6

Zein, Laila Fariha, \& Setiawan, Adib Rifqi. (2019, April 9). Women's Beauty in As If It's Your Last by BLACKPINK. Frenxiv. DOI: https://doi.org/10.31226/osf.io/57n8t

Zein, Laila Fariha, \& Setiawan, Adib Rifqi. (2019, March 19). Kajian Semiotika Terhadap Lagu Julia Perez. INA-Rxiv. DOI: https://doi.org/10.31227/osf.io/pevgb

Zein, Laila Fariha, \& Setiawan, Adib Rifqi. (2019, March 19). Melihat Tempat Perempuan dalam kitab Kuning. INA-Rxiv. DOI: https://doi.org/10.31227/osf.io/c96up

Zein, Laila Fariha, \& Setiawan, Adib Rifqi. (2019, March 19). Salahkah Menjadi Perempuan Cantik?. INA-Rxiv. DOI: https://doi.org/10.31227/osf.io/65bdt 